\title{
Molecular cytogenetic identification of a wheat (Triticum aestivum)-American dune grass (Leymus mollis) translocation line resistant to stripe rust
}

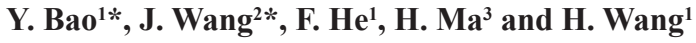 \\ ${ }^{1}$ Agronomy College, State Key Laboratory of Crop Science, \\ Shandong Key Laboratory of Crop Science, \\ Tai' an Subcenter of National Wheat Improvement Center, \\ Shandong Agricultural University, Tai'an, China \\ ${ }^{2}$ Jining Institute of Agricultural Sciences, Jining, China \\ ${ }^{3}$ Zaozhuang Institute of Agricultural Sciences, Zaozhuang, China \\ *These authors contributed equally to this study. \\ Corresponding author: H. Wang \\ E-mail: hgwang@sdau.edu.cn
}

Genet. Mol. Res. 11 (3): 3198-3206 (2012)

Received August 25, 2011

Accepted January 23, 2012

Published May 22, 2012

DOI http://dx.doi.org/10.4238/2012.May.22.2

\begin{abstract}
Leymus mollis, a perennial allotetraploid $(2 \mathrm{n}=4 \mathrm{x}=28)$, known as American dune grass, is a wild relative of wheat that could be useful for cultivar improvement. Shannong0096, developed from interspecific hybridization between common wheat $\mathrm{cv}$. Yannong15 and $L$. mollis, was analyzed with cytological procedures, genomic in situ hybridization, stripe-rust resistance screening and molecular marker analysis. We found that Shannong0096 has 42 chromosomes in the root-tip cells at mitotic metaphase and 21 bivalents in the pollen mother cells at meiotic metaphase I, demonstrating cytogenetic stability. Genomic in situ hybridization probed with total genomic DNA from $L$. mollis gave strong hybridization signals in the distal
\end{abstract}


region of two wheat chromosome arms. A single dominant $Y r$ gene, derived from L. mollis and temporarily designated as $\mathrm{YrSn0096}$, was found on the long arm of chromosome 4A of Shannong0096. YrSn0096 should be a novel $\mathrm{Yr}$ gene because none of the previously reported $\mathrm{Yr}$ genes on chromosome 4A are related to L. mollis. This gene was found to be closely linked to the loci Xbarc236 and Xksum134 with genetic distances of 5.0 and $4.8 \mathrm{cM}$, respectively. Based on data from $267 \mathrm{~F}_{2}$ plants of Yannong15/Huixianhong, the linkage map of $Y r S n 0096$, using the two molecular markers, was established in the order Xbarc236-YrSn0096-Xksum134. Shannong0096 appeared to be a unique wheat- L. mollis translocation with cryptic alien introgression. Cytogenetic stability, a high level of stripe-rust resistance, the common wheat background, and other positive agronomic traits make it a desirable donor for introducing novel alien resistance genes in wheat breeding programs, with the advantage of molecular markers that can be used to confirm introgression.

Key words: Genomic in situ hybridization; Leymus mollis; Stripe rust; Translocation

\section{INTRODUCTION}

As the second most frequently planted crop, wheat (Triticum aestivum L.) plays an important role in food security for the world's population. More than $75 \%$ of the world's population is estimated to consume wheat as part of the daily diet (Afzal et al., 2008). To meet the needs of the growing world population, the demand for wheat is expected to grow faster than that for any other major agricultural crop (Dreisigacher, 2004). However, wheat production is hampered by many diseases.

Stripe rust (syn. yellow rust), caused by Puccinia striiformis Westend. f. sp tritici Eriks, is one of the most devastating fungal diseases of wheat in many cool and temperate regions (Wan et al., 2007). In recent years, this disease has become increasingly important in some wheat-growing areas, where it had previously been absent or not regularly destructive (Zhang et al., 2009). Stripe rust inflicts not only yield losses - ranging from 10 to $70 \%$ and, in some cases, up to $100 \%$ - but also quality downgrades (Chen, 2005). The application of fungicides has been a preferred method for the rapid and effective control of stripe rust. It can also cause large expenditures, contribute to serious environmental and health problems, and result in the selection of fungicide-resistant strains of the pathogen, however (Chen, 2005; Cao et al., 2008). Breeding resistant cultivars has been considered the most effective, economical, and environmentally acceptable approach to stripe rust control (Chen, 2005; Cao et al., 2008; Sui et al., 2009; Hu et al., 2011). To date, more than 50 officially named $Y r$ genes at 49 loci (Yrl-Yr49) and some temporarily designated genes have been documented (the Komugi Wheat Genetic Resources Database, seeing the reference $a$ for the URL [http://www.shigen.nig.ac.jp/wheat/ komugi/genes/symbolListPageAction.do?page=-1]). Most of these genes are race specific, and virulences to them have been detected in various parts of the world (Li et al., 2011). Consequently, many $Y r$ genes have lost their resistance to the fungus (Kang et al., 2010), necessitating 
a constant search for and transfer of new and effective stripe rust resistance genes.

One reality is that the replacement of highly variable land races by high-yielding, pure-line varieties in many parts of the world has narrowed the genetic base for disease resistance in the wheat gene pool (Kuraparthy et al., 2007a). Fortunately, relatives of wheat have been found to be invaluable sources of disease-resistance genes (Jiang et al., 1994; Friebe et al., 1996). So far, an increasing number of stripe rust resistance genes originating in the wild relatives of wheat have been identified, such as $Y r 9$ from Secale cereale (the Komugi Wheat Genetic Resources Database, seeing the reference $b$ for the URL [http://www.shigen.nig.ac.jp/ wheat/komugi/genes/symbolDetailAction.do?geneId=1637]), Yr28 from Aegilops tauschii (Singh et al., 2000), and Yr40 from Aegilops geniculata (Kuraparthy et al., 2007a).

Leymus mollis (Trin.) Hara, a perennial allotetraploid $(2 \mathrm{n}=4 \mathrm{x}=28)$, is an invaluable wild relative for wheat improvement because of its many superior agronomic characters such as disease resistance, saline-alkali tolerance, exceptionally large spikes with numerous floscules, and vigorous growth (Wang et al., 2000; Kishii et al., 2003). The distant relationship presents obstacles to obtaining hybrids between L. mollis and wheat, however. In the 1980s, Fu et al. (1993) acquired the first wheat-L. mollis hybrids through embryo rescue and subsequently created alien chromosome lines, including partial amphidiploids, additions, and substitutions, with varying positive traits derived from L. mollis (Fu et al., 1996, 1997). Few reports are available, however, on wheat- $L$. mollis translocations, the most valuable germplasms transferring beneficial genes from L. mollis to common wheat. Line Shannong0096, derived from the progeny of a wheat- $L$. mollis octoploid and bread wheat cv. Yannong15, displays excellent resistance to stripe rust as well as other favorable agronomic characteristics such as numerous tillers and vigorous growth. Herein, we report the cytogenetic identification of Shannong0096 and molecular analysis of its stripe rust resistance.

\section{MATERIAL AND METHODS}

\section{Plant materials}

Plant materials used in this study included L. mollis, a wheat-L. mollis octoploid, Shannong0096, and wheat varieties Chinese Spring, Yannong15, and Huixianhong. An $\mathrm{F}_{2}$ segregation population was construed on the basis of the cross between Shannong0096 and Huixianhong (highly susceptible to stripe rust). L. mollis and the wheat-L. mollis octoploid were provided by Professor Jie Fu, retired from the Northwest Institution of Botany, Chinese Academy of Sciences, Yangling, China. Shannong0096 was developed from the progeny of Yannong15 and the wheat-L. mollis octoploid at the Agronomy College of Shandong Agricultural University, Tai'an, China. All plant materials were preserved via selfing at the Tai'an Subcenter of the National Wheat Improvement Center, Shandong, China.

\section{Cytogenetic identification}

Seeds were germinated at $25^{\circ} \mathrm{C}$ on moist filter paper on Petri dishes, maintained at $4^{\circ} \mathrm{C}$ for approximately $24 \mathrm{~h}$, and then returned to $25^{\circ} \mathrm{C}$. Roots $(1-2 \mathrm{~cm})$ were cut and immersed in ice water for approximately $24 \mathrm{~h}$ before fixing in Carnoy's solution. After fixation, the root tips were stained and flattened in carbol fuchsin, and mitotic chromosomes were observed un- 
der a microscope. When plants attained the flag-leaf stage, spikes were sampled, and anthers at metaphase I of meiosis were fixed in Carnoy's solution, dissociated in $1 \mathrm{M} \mathrm{HCl}$ at $60^{\circ} \mathrm{C}$ for 6-8 min, and flattened in 1\% acetocarmine.

For genomic in situ hybridization (GISH), the total genomic DNA from L. mollis was labeled with digoxigenin-11-2'-deoxyuridine-5'-triphosphate with the nick translation method and used as a probe. Sheared genomic DNA from Chinese Spring wheat (ABD genomes, $2 \mathrm{n}=42$ ) was used as blocking DNA. Detailed procedures of chromosome preparation and hybridization mixture are described elsewhere (Chen et al., 1998). The GISH signals were detected with fluorescein-conjugated anti-digoxigenin antibodies, and the slides were mounted in Vectashield antifade solution containing propidium iodide. Photographs were taken with a fluorescence microscope equipped with a chargecoupled device camera.

\section{Stripe rust screening}

A mixture of $P$. striiformis races, including Chinese Yellow Rust 29, Chinese Yellow Rust 31, Chinese Yellow Rust 32, Shuiyuan11, Shuiyuan12, Shuiyuan13, and Shuiyuan14, was used to evaluate resistance to stripe rust. Shannong0096, Yannong15, L. mollis, the wheat- $L$. mollis octoploid, and the $\mathrm{F}_{2}$ population of Shannong0096/Huixianhong were planted along with Huixianhong at the Research Farm of Shandong Agricultural University, Tai'an $\left(116^{\circ} 20^{\prime} \sim 117^{\circ} 59^{\prime}\right.$ E, $\left.35^{\circ} 38^{\prime} \sim 36^{\circ} 28^{\prime} \mathrm{N}\right)$, Shandong, China. Artificial inoculation was carried out twice with the mixed races at the jointing stage and 7 days later. The infection types (ITs) were classified 21 days after inoculation according to a 0 to 4 scale (Bariana and McIntosh, 1993), on which 0 indicated the presence of no visible symptoms or no signs of infection or necrotic flecks, 1 indicated the presence of necrotic and chlorotic areas with restricted sporulation, 2 indicated the presence of moderate sporulation with necrosis and chlorosis, 3 indicated the presence of sporulation with chlorosis, and 4 indicated the presence of abundant sporulation without chlorosis. Plants with ITs of 0-2 were considered resistant, and those with ITs of 3-4 were deemed susceptible.

\section{Microsatellite screening and electrophoretic analysis}

Microsatellite primers, designated as either simple sequence repeats (SSRs) or expressed sequence tag-derived SSRs (EST-SSRs), were used to scan the plant materials for useful polymorphisms. SSR primers were synthesized following the sequences published in the GrainGenes database (http://wheat.pw.usda.gov), and the EST-SSR primers were provided by Professor Sishen Li, Agronomy College of Shandong Agricultural University, Tai'an, China. Each $25-\mu \mathrm{L}$ polymerase chain reaction (PCR) solution contained $2.5 \mu \mathrm{L}$ 10X PCR buffer, 2.0 $\mu \mathrm{L} \mathrm{Mg}^{2+}(2.5 \mathrm{mM}), 1.5 \mu \mathrm{L}$ deoxynucleotide triphosphates $(2.5 \mathrm{nM}), 1.0 \mathrm{U}$ Taq polymerase, $3.0 \mu \mathrm{L}$ primer pair $(5 \mu \mathrm{M})$, and $3.0 \mu \mathrm{L}$ DNA $(30 \mathrm{ng} / \mu \mathrm{L})$. DNA amplification was carried out in a GeneAmp PCR System 9600 programmed for $4 \mathrm{~min}$ at $94^{\circ} \mathrm{C}$ for predenaturation and 35 cycles each consisting of $45 \mathrm{~s}$ at $94^{\circ} \mathrm{C}, 30 \mathrm{~s}$ at $50^{\circ}-60^{\circ} \mathrm{C}$ (depending on the primers), and $1 \mathrm{~min}$ at $72^{\circ} \mathrm{C}$, followed by a final extension for $10 \mathrm{~min}$ at $72^{\circ} \mathrm{C}$. The PCR-amplified fragments were separated on a $6 \%$ nondenaturing polyacrylamide gel, stained with silver nitrate, and visualized using a Tanon Gis-2010 gel-imaging system. 


\section{Data analysis}

Chi-square tests were used to evaluate the goodness of fit of observed and expected ratios for stripe rust reactions and molecular markers. The linkage relationship between the markers and the resistance gene was analyzed using the Mapmaker/Exp software, Version 3.0.

\section{RESULTS}

\section{Cytological identification of Shannong0096}

Chromosome counts and meiotic observation of pollen mother cells in 15 Shannong0096 plants revealed the presence of 42 chromosomes in the root-tip cells (Figure 1A) and 21 bivalents at meiotic metaphase I (Figure 1B) indicative of the cytogenetic stability of Shannong0096. Using digoxigenin-labeled total genomic DNA from L. mollis as a probe and Chinese Spring DNA as a blocker, GISH was carried out to detect the alien genetic materials in Shannong0096. In mitotic metaphase cells, two chromosomes were labeled green in the terminal regions and the others were counterstained red (Figure 1C), indicating that Shannong0096 was a translocation line with a small fragment from L. mollis translocated to the distal region of a wheat chromosome.

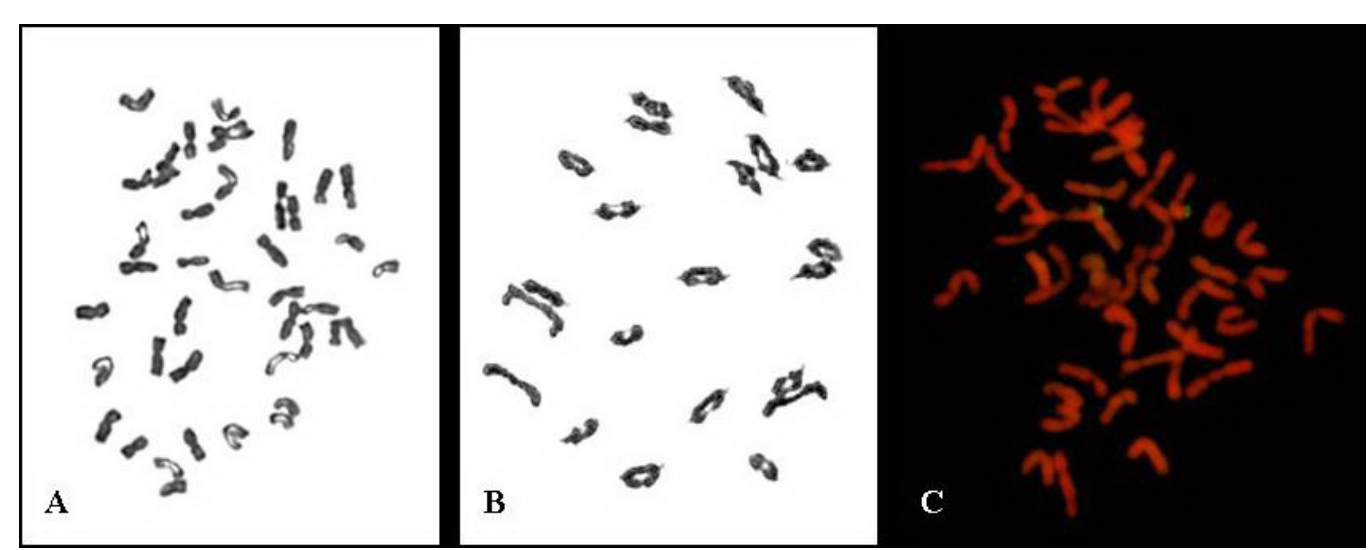

Figure 1. Cytological patterns of Shannong0096. A. A root-tip cell at mitotic metaphase $(2 n=42)$. B. A pollen mother cell at meiotic metaphase I $(2 \mathrm{n}=21 \mathrm{II})$. C. A genomic in situ hybridization pattern probed with total genomic DNA from Leymus mollis.

\section{Reactions to stripe rust}

After inoculation with stripe rust isolates, both the parent Yannong 15 and the control Huixianhong were susceptible, whereas Shannong0096, L. mollis, and the wheat-L. mollis octoploid were immune (Table 1). In addition, all the wheat parents of the wheat- L. mollis octoploid-namely, 7182-0-11-1, Dasui78-3, and Zhi763-were susceptible (Zhou et al., 2001). Therefore, the pedigree persuaded us to conclude that $\mathrm{YrSnO096}$ originated from L. mollis. 
Table 1. Reactions of Huixianhong, Shannong0096 and their parents to stripe rust.

\begin{tabular}{|c|c|c|c|c|c|}
\hline & Leymus mollis & Wheat-L. mollis octoploid & Yannong15 & Shannong0096 & Huixianhong \\
\hline Material type & Donor & Donor & Receptor & Translocation & Control \\
\hline Infection types & 0 & 0 & $3 \sim 4$ & $0 \sim 0$; & 4 \\
\hline
\end{tabular}

\section{Genetic analysis of stripe rust resistance in Shannong0096}

To determine the inheritance of the resistance gene(s) in Shannong0096, we developed an $\mathrm{F}_{2}$ population derived from the cross Shannong0096/Huixianhong. After infection by the mixed races of stripe rust, these plants showed apparent symptoms of reactions related to resistance or susceptibility in a resistant:susceptible ratio of 3:1 (Table 2). Thus, stripe rust resistance in Shannong0096 indicated a typical dominant inheritance proved to be controlled by a single gene or locus, which was temporarily designated $\mathrm{YrSn0096.}$

\begin{tabular}{|c|c|c|c|}
\hline & No. of $F_{2}$ plants investigated & Expected ratio & $\chi^{2}$ \\
\hline$F_{2}$ plants of Shannong0096/Huixianghong & $289 \mathrm{R}$ & $3: 1$ & 0.4268 \\
\hline
\end{tabular}

$\mathrm{R}=$ resistant; $\mathrm{S}=$ susceptible.

\section{Molecular linkage for $\mathrm{YrSn0096}$}

Of the 1261 SSR and EST-SSR primer pairs used for screening, Xbarc236 ${ }_{255}$ and Xksum 134 ${ }_{245}$ were identified as potential linkage markers for $\mathrm{YrSn0096.} \mathrm{For} \mathrm{instance,} \mathrm{the}$ primer BARC236 generated two special bands (Figure 2): one present in susceptible or resistant parents, designated as $X b a r c 236_{250}$, and the other present only in resistant parents, designated $X_{\text {barc } 236_{255}}$. The resistant $\mathrm{F}_{2}$ plants amplified Xbarc236 ${ }_{255}$ (homozygous) or both $X$ barc $236_{250}$ and $X$ barc $236_{255}$ (heterozygous), whereas the susceptible bulk and the susceptible $\mathrm{F}_{2}$ individuals amplified only the $B A R C 236_{250}$ band.

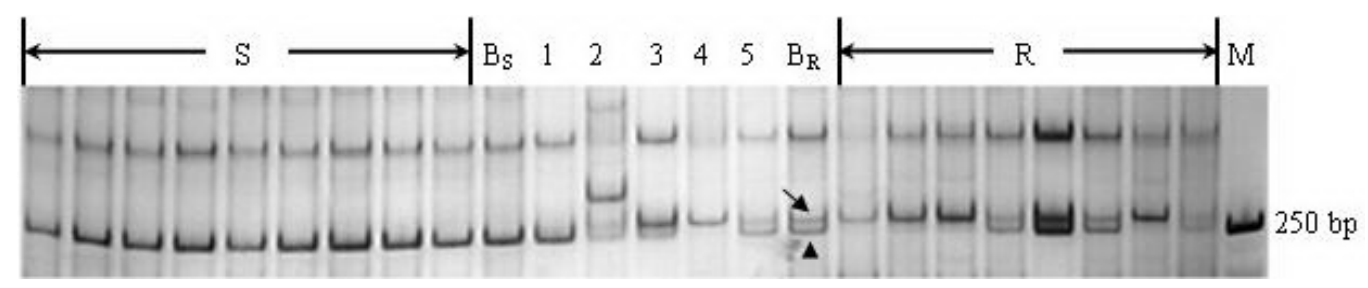

Figure 2. Electrophoresis of PCR products amplified with SSR marker Xbarc236-4A on a polyacrylamide gel. Lane 1 = Huixianhong; lane 2 = Yannong15; lane 3 = Shannong0096; lane $4=$ Wheat- $L$. mollis octoploid; lane $5=L$. mollis; lane $S=$ susceptible $\mathrm{F}_{2}$; lane $R=$ resistant $\mathrm{F}_{2}$; lane $M=$ molecular marker.

To obtain genetic data, we further analyzed the genotypes of the loci Xbarc236 and Xksum 134 in $267 \mathrm{~F}_{2}$ individuals (Table 3). In the case of Xbarc236, of 199 resistant plants,

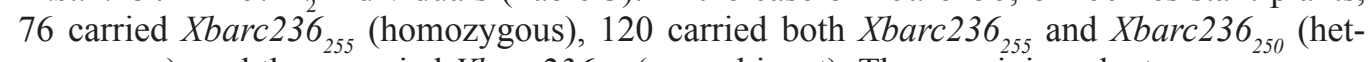
erozygous), and three carried $X b a r c 236_{250}$ (recombinant). The remaining plants were suscep- 
tible and carried $X b a r c 236_{250}$. Therefore, the $\mathrm{F}_{2}$ genotypes segregated in a 1AA:2Aa:1aa ratio, which confirmed the involvement of a single gene in their inheritance of resistance. The same pattern was observed in the EST-SSR marker Xksum 134. Using the Mapmaker software, we found the linkage relationship of Xbarc236 and Xksum134 with YrSn0096 (Figure 3). The two loci Xbarc236 and Xksum 134 were genetically associated with $\mathrm{YrSn0096}$, with distances of 5.0 and $4.8 \mathrm{cM}$, respectively. Based on the published chromosomal location of Xbarc236 (the GrainGenes Database, seeing the reference $c$ for the URL [http://wheat.pw.usda.gov/cgibin/graingenes/report.cgi?class=locus;name $=$ Xbarc236-4A]) and Xksum134 (Yu et al., 2004), YrSn0096 was deduced to be located on the long arm of wheat chromosome 4A.

\begin{tabular}{|c|c|c|c|c|c|c|c|}
\hline \multirow[t]{2}{*}{ Markers } & \multirow[t]{2}{*}{ Phenotypes } & \multicolumn{3}{|c|}{ Genotypes } & \multirow[t]{2}{*}{ Total } & \multirow[t]{2}{*}{ Expected ratio } & \multirow[t]{2}{*}{$\chi^{2}$} \\
\hline & & AA & $\mathrm{Aa}$ & aa & & & \\
\hline \multirow[t]{3}{*}{ Xbarc236-4A } & $\mathrm{R}$ & 76 & 120 & 3 & 199 & \multirow[t]{3}{*}{$1: 2: 1$} & \multirow[t]{3}{*}{2.92} \\
\hline & S & 0 & 0 & 68 & 68 & & \\
\hline & Total & 76 & 120 & 71 & 267 & & \\
\hline \multirow[t]{3}{*}{ Xksum134 } & $\mathrm{R}$ & 75 & 118 & 6 & 199 & \multirow[t]{3}{*}{$1: 2: 1$} & \multirow[t]{3}{*}{2.92} \\
\hline & S & 1 & 2 & 65 & 68 & & \\
\hline & Total & 76 & 120 & 71 & 267 & & \\
\hline
\end{tabular}

$\mathrm{R}=$ resistant; $\mathrm{S}=$ susceptible.

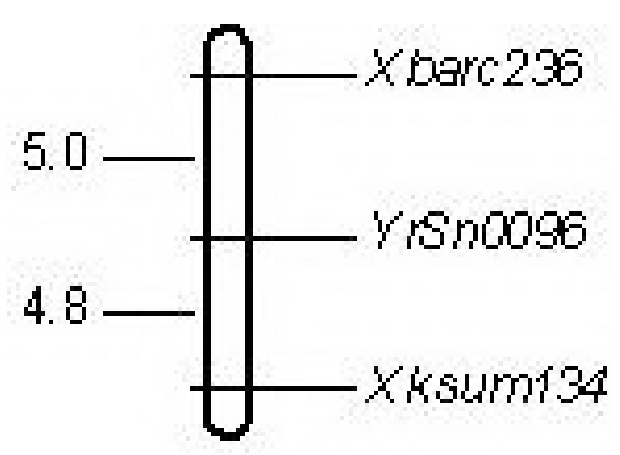

Figure 3. Linkage map of the stripe-rust resistance gene $Y r S n 0096$, flanked by two markers on the wheat chromosome 4AL.

\section{DISCUSSION}

Wild relatives and related species are valuable reservoirs for broadening the genetic variability of common wheat. Generally, the first step for transferring exotic genes is the production of an amphidiploid (or partial amphidiploid) between wheat and the alien species, followed by the production of chromosome addition or substitution lines, and finally, the production of translocation lines. Producing these lines is an enormous and time-consuming chore. Moreover, many derivatives of these wheat-alien species lose the resistance derived from alien species at a high rate (Cao et al., 2008) owing to the cytological instability of alien chromosome segments incorporated into non-homoeologous regions (Nasuda et al., 1998). Shannong0096 was developed directly from the progeny of a wheat-L. mollis octoploid and 
Yannong15 wheat, the latter used as the recurrent parent. The present study demonstrates its cytological stability and excellent resistance to stripe rust, suggesting the feasibility of transferring alien genes to wheat with genetic stability directly by crossing and backcrossing.

Genetic information, including inheritance, type, and number of resistance genes, could be used to predict the durability of resistance in breeding lines and commercial cultivars (Sui et al., 2009). In the present study, we identified a stripe rust resistance gene, YrSn0096, in the wheat-L. mollis translocation line Shannong0096. Similar to many reported $Y r$ genes, $\mathrm{YrSn0096}$ is controlled by a single, dominant locus. It resists prevailing stripe rust races, including the predominant race Chinese Yellow Rust 32, which caused resistance loss in nearly all Chinese wheat cultivars and, consequently, a large epidemic of the fungus in China in 2002. We localized this gene to the long arm of wheat chromosome 4A using the markers BARC236 and KSUM134. The previously reported $\mathrm{Yr}$ genes $\mathrm{YrHVII}$, YrMin, and $\mathrm{YrND}$ are also located on this chromosome (the Komugi Wheat Genetic Resources Database, seeing the reference $a$ for the URL), but none of them is related to L. mollis. Therefore, $\mathrm{YrSn} 0096$ must be a novel $\mathrm{Yr}$ gene. Compared to the incorporation of other exotic sources, incorporating the high-resistance gene $\mathrm{YrSn0096}$ into different wheat cultivars should be relatively easy owing to the common wheat background and the many other favorable agronomic traits of Shannong0096.

Recently, researchers have identified a few disease-resistant alien translocation lines with cytologically undetectable alien segments, which were called "cryptic alien introgressions" (Kuraparthy et al., 2007b; He et al., 2009; Luo et al., 2009). In the present study, two markers flanked $\mathrm{YrSn0096}$ on the long arm of chromosome 4A, whereas GISH mapped a small alien segment to the short arm of a wheat chromosome. It suggested that Shannong0096 has two alien segments: one detected by GISH in the distal region of the short arm of a wheat chromosome that did not carry $\mathrm{YrSn0096}$, and the other a cryptic introgression located on $4 \mathrm{AL}$ carrying the resistance gene. The presence of these alien segments is probably the reason for the seeming inconsistency between the GISH results and the microsatellite data.

\section{ACKNOWLEDGMENTS}

Research supported by the National Natural Science Foundation of China (\#30771349, \#30971765).

\section{REFERENCES}

Afzal SN, Haque MI, Ahmedani MS, Rauf A, et al. (2008). Impact of stripe rust on kernel weight of wheat varieties sown in rainfed areas of Pakistan. Pak. J. Bot. 40: 923-929.

Bariana HS and McIntosh RA (1993). Cytogenetic studies in wheat. XV. Location of rust resistance genes in VPM1 and their genetic linkage with other disease resistance genes in chromosome 2A. Genome 36: 476-482.

Cao Z, Deng Z, Wang M, Wang X, et al. (2008). Inheritance and molecular mapping of an alien stripe-rust resistance gene from a wheat-Psathyrostachys huashanica translocation line. Plant Sci. 174: 544-549.

Chen Q, Conner RL, Ahmad F, Laroche A, et al. (1998). Molecular characterization of the genome composition of partial amphiploids derived from Triticum aestivum $\times$ Thinopyrum ponticum and T. aestivum $\times$ Th. intermedium as sources of resistance to wheat streak mosaic virus and its vector, Aceria tosichella. Theor. Appl. Genet. 97: 1-8.

Chen XM (2005). Epidemiology and control of stripe rust [Puccinia striiformis f. sp. tritici] on wheat. Can. J. Plant. Pathol. 27: 314-337.

Dreisigacker S (2004). Genetic Diversity in Elite Lines and Land Races of CIMMYT Spring Bread Wheat and Hybrid Performance of Crosses Among Elite Germplasm. Ph.D. thesis, Faculty of Agriculture, University of Hohenheim, Hohenheim. 
Friebe B, Jiang J, Raupp WJ, McIntosh RA, et al. (1996). Characterization of wheat-alien translocations conferring resistance to diseases and pests: current status. Euphytica 91: 59-87.

Fu J, Chen S and Zhang A (1993). Studies of the formation and cytogenetics of octoploid Tritileymus. Acta Genet. Sin. 20: 317-323.

Fu J, Chen S, Zhang A, Hou W, et al. (1996). Cytogenetic studies on the cross progenies between octoploid Tritileymus and Triticum aestivum. Acta Genet. Sin. 23: 24-31.

Fu J, Xu X, Yang Q, Chen S, et al. (1997). Cytogenetic studies on the cross between octoploid Tritileymus and nulllisomic wheat. Acta Genet. Sin. 24: 350-357.

He R, Chang Z, Yang Z, Yuan Z, et al. (2009). Inheritance and mapping of powdery mildew resistance gene $P m 43$ introgressed from Thinopyrum intermedium into wheat. Theor. Appl. Genet. 118: 1173-1180.

Hu LJ, Li GR, Zeng ZX, Chang ZJ, et al. (2011). Molecular cytogenetic identification of a new wheat-Thinopyrum substitution line with stripe rust resistance. Euphytica 177: 169-177.

Jiang J, Friebe B and Gill BS (1994). Recent advances in alien gene transfer in wheat. Euphytica 73: 199-212.

Kang Z, Zhao J, Han D, Zhang H et al. (2010). Status of wheat rust research and control in China. Available at [http:// www.globalrust.org/db/attachments/bgriiwc/24/2/07-kang-ca-A4-embargo.pdf].

Kishii M, Wang RR and Tsujimoto H (2003). Characteristics and behaviour of the chromosomes of Leymus mollis and $L$. racemosus (Triticeae, Poaceae) during mitosis and meiosis. Chromosome Res 11: 741-748.

Kuraparthy V, Chhuneja P, Dhaliwal HS, Kaur S, et al. (2007a). Characterization and mapping of cryptic alien introgression from Aegilops geniculata with new leaf rust and stripe rust resistance genes Lr57 and $\mathrm{Yr} 40$ in wheat. Theor. Appl. Genet. 114: 1379-1389.

Kuraparthy V, Sood S, Chhuneja P, Dhaliwal HS, et al. (2007b). A cryptic wheat-Aegilops triuncialis translocation with leaf rust resistance gene Lr58. Crop Sci. 47: 1995-2003.

Li Q, Chen XM, Wang MN and Jing JX (2011). Yr45, a new wheat gene for stripe rust resistance on the long arm of chromosome 3D. Theor. Appl. Genet. 122: 189-197.

Luo PG, Luo HY, Chang ZJ, Zhang HY, et al. (2009). Characterization and chromosomal location of Pm40 in common wheat: a new gene for resistance to powdery mildew derived from Elytrigia intermedium. Theor. Appl. Genet. 118: 1059-1064.

Nasuda S, Friebe B, Busch W, Kynast RG, et al. (1998). Structural rearrangement in chromosome 2M of Aegilops comosa has prevented the utilization of the compair and related wheat-Ae. comosa translocations in wheat improvement. Theor. Appl. Genet. 96: 780-785.

Singh RP, Nelson JC and Sorrells ME (2000). Mapping Yr28 and other genes for resistance to stripe rust in wheat. Crop Sci. 40: 1148-1155.

Sui XX, Wang MN and Chen XM (2009). Molecular mapping of a stripe rust resistance gene in spring wheat cultivar Zak. Phytopathology 99: 1209-1215.

Wan AM, Chen XM and He ZH (2007). Wheat stripe rust in China. Aust. J. Agr. Res. 58: 605-619.

Wang XP, Fu J, Zhang XQ, Jing JK, et al. (2000). Molecular cytogenetic study on genome constitutions of octoploid Tritileymus. Acta Bot. Sin. 42: 582-586.

Yu JK, Dake TM, Singh S, Benscher D, et al. (2004). Development and mapping of EST-derived simple sequence repeat markers for hexaploid wheat. Genome 47: 805-818.

Zhang P, McIntosh RA, Hoxha S and Dong C (2009). Wheat stripe rust resistance genes $\mathrm{Yr} 5$ and $\mathrm{Yr} 7$ are allelic. Theor. Appl. Genet. 120: 25-29.

Zhou YC, Zhang XQ, Wang XP, Wu LR, et al. (2001). Chromosomal location and molecular marker of resistance gene to Puccinia striiformis west. in Leymus mollis Trin. Hara. Yi Chuan Xue Bao 28: 864-869. 